\title{
Dark matter physics in dark $S U(2)$ gauge symmetry with non-Abelian kinetic mixing
}

\author{
P. Ko๑, ${ }^{1,2, *}$ Takaaki Nomura, ${ }^{1, \dagger}$ and Hiroshi Okada ${ }^{3, *}$ \\ ${ }^{1}$ School of Physics, KIAS, Seoul 02455, Korea \\ ${ }^{2}$ Quantum Universe Center, KIAS, Seoul 02455, Korea \\ ${ }^{3}$ Asia Pacific Center for Theoretical Physics (APCTP)_Headquarters San 31, \\ Hyoja-dong, Nam-gu, Pohang 790-784, Korea
}

(Received 10 November 2020; accepted 17 April 2021; published 18 May 2021)

\begin{abstract}
We investigate a model of dark sector based on non-Abelian $S U(2)_{D}$ gauge symmetry. This dark gauge symmetry is broken into discrete $Z_{2}$ via vacuum expectation values of two real triplet scalars, and an $S U(2)_{D}$ doublet Dirac fermion becomes $Z_{2}$-odd particles whose lighter component makes stable dark matter candidate. The standard model and dark sector can be connected via the scalar mixing and the gauge kinetic mixing generated by higher dimensional operators. We then discuss relic density of dark matter and implications to collider physics in the model. The most unique signatures of this model at the LHC would be the dark scalar $\left(\Phi_{1}^{\left({ }^{\prime}\right)}\right)$ productions where it subsequently decays into: (1) a fermionic dark matter $\left(\chi_{l}\right)$ and a heavy dark fermion $\left(\chi_{h}\right)$ pair, $\Phi_{1}^{\left({ }^{\prime}\right)} \rightarrow \bar{\chi}_{l} \chi_{h}\left(\bar{\chi}_{h} \chi_{l}\right)$, followed by $\chi_{h}$ decays into $\chi_{l}$ and non-Abelian dark gauge bosons $\left(X_{i}\right.$ 's) which decays into SM fermion pair $\bar{f}_{\mathrm{SM}} f_{\mathrm{SM}}$ resulting in the reaction $p p \rightarrow \Phi_{1}^{\left({ }^{\prime}\right)} \rightarrow \bar{\chi}_{h} \chi_{l}\left(\bar{\chi}_{l} \chi_{h}\right) \rightarrow f_{\mathrm{SM}} \bar{f}_{\mathrm{SM}} \chi_{l} \bar{\chi}_{l}$, (2) a pair of $X_{i}$ 's followed by $X_{i}$ decays into a DM pair or the SM fermions resulting in the reaction, $p p \rightarrow \Phi_{1}^{\left({ }^{\prime}\right)} \rightarrow X_{i} X_{i} \rightarrow \bar{\chi}_{l} \chi_{l} f_{\mathrm{SM}} \bar{f}_{\mathrm{SM}}$ or even number of $f_{\mathrm{SM}} \bar{f}_{\mathrm{SM}}$ pairs.
\end{abstract}

DOI: 10.1103/PhysRevD.103.095011

\section{INTRODUCTION}

The standard model (SM) of particle physics has been very successful in describing phenomenology observed in various experiments. However the existence of dark matter (DM) cannot be explained in the SM framework, and it would be described as a new particle associated with physics beyond the SM. The nature of DM is an open question and there are many experimental searches for interactions among DM and the SM particles such as in direct detection, indirect detection, and collider experiments. No clear evidence of DM would indicate a dark sector which is hidden from current observations.

As the SM is described by local gauge symmetries, it is plausible that the dark sector is also ruled by a hidden/dark gauge symmetry. Moreover stability of DM indicates necessity of a symmetry to protect it from decay, and it

\footnotetext{
*pko@kias.re.kr

nomura@kias.re.kr

"hiroshi.okada@apctp.org
}

Published by the American Physical Society under the terms of the Creative Commons Attribution 4.0 International license. Further distribution of this work must maintain attribution to the author(s) and the published article's title, journal citation, and DOI. Funded by SCOAP . can be a remnant of dark gauge symmetry (see Ref. [1] for a review along this line). Thus, it is an attractive scenario that dark gauge symmetry is spontaneously broken to a symmetry stabilizing DM candidate. To realize this concept, we are especially interested in the extension of the SM introducing a new $S U(2)_{D}$ gauge symmetry where all the SM fields are singlet under it. The interesting properties of a model with local $S U(2)_{D}$ group is that an unbroken discrete symmetry can be naturally preserved after the spontaneous breaking of the $S U(2)_{D}$ gauge symmetry comparing with a hidden local $U(1)$ case in which the $U(1)$ charge has to satisfy some artificial tuning [2]. For example, if we require remnant $Z_{2}$ symmetry from local $U(1)$ the charge of fields should be integer with spontaneous symmetry breaking by scalar field having even charge; then particles with even(odd) charge become $Z_{2}$ even(odd). Thus we need to tune charge of fields to get discrete symmetry while any real number is possible for value of charges as long as anomaly free. On the other hand, we have less choice for representation of nonAbelian gauge symmetry considering fundamental and adjoint representations, and one can say it is more natural if we get discrete symmetry from the non-Abelian case. Note that various applications of the hidden $S U(2)$ gauge symmetry have been studied in literatures, for examples, a remaining $Z_{2}$ symmetry with triplet scalar [3], a remaining 
$Z_{3}\left(Z_{4}\right)$ symmetry with a quadruplet(quintet) in Refs. [4-7], $Z_{2} \times Z_{2}^{\prime}$ symmetry [8], a custodial symmetry in Refs. [9,10], an unbroken $U(1)$ from $S U(2)$ in Refs. [11-13], a model adding hidden $U(1)_{h}[14]$ and a model with classical scale invariance [15].

In this paper, we discuss a minimal fermion DM model with local $S U(2)_{D}$ breaking into discrete $Z_{2}$ symmetry where we introduce two $S U(2)_{D}$ triplet real scalar fields $(\vec{\phi}$ and $\vec{\phi}^{\prime 1}$ ) developing vacuum expectation values (VEVs) and an $S U(2)_{D}$ doublet Dirac fermion $\chi$ as $Z_{2}$-odd DM candidate, ${ }^{2}$ and analyze its phenomenology such as DM relic density and collider physics. We also introduce higher dimensional operators that induce gauge kinetic mixing terms between $S U(2)_{D}$ and $U(1)_{Y}$ gauge fields after $S U(2)_{D}$ symmetry breaking by nonzero VEV's of $\vec{\phi}$ and $\vec{\phi}^{\prime}$ as mediators between the dark gauge sector and the SM sector. After fixing our model, we formulate particle mass spectra and their interactions in the dark sector and the portals to the SM sector. Then relic density of our DM candidate is estimated scanning free parameters globaly where we also take into account constraint from direct detection of DM. Furthermore we discuss implications to collider physics considering the scalar portal and the kinetic mixing as connections between dark sector and the SM.

This paper is organized as follows. In Sec. II, we show our model of $S U(2)_{D}$ dark sector formulating mass spectra and interactions. In Sec. III, we analyze DM relic density and discuss the allowed parameter region. In Sec. IV, we discuss implications to collider physics. Finally, we conclude and discuss in Sec. V.

\section{MODEL AND FORMULAS}

In this section we summarize the setup for our model. We introduce a dark sector which is controlled by a nonAbelian $S U(2)_{D}$ dark gauge symmetry, with two real scalar fields $\vec{\phi}$ and $\vec{\phi}^{\prime}$, and one Dirac fermion $\chi$ as summarized in Table I. In components, $\vec{\phi}\left(\vec{\phi}^{\prime}\right)$ and $\chi$ are written as

$$
\vec{\phi}\left[\vec{\phi}^{\prime}\right]=\left(\phi_{1}\left[\phi_{1}^{\prime}\right], \phi_{2}\left[\phi_{2}^{\prime}\right], \phi_{3}\left[\phi_{3}^{\prime}\right]\right), \quad \chi=\left(\chi_{1}, \chi_{2}\right)^{T},
$$

where the indices for triplet scalars correspond to three $S U(2)_{D}$ generators.

The $S U(2)_{D}$ dark gauge symmetry is spontaneously broken by nonzero VEV's of two real scalar triplets $\phi$ and $\phi^{\prime}$. In our scenario, we assume VEV alignments of two scalar triplets as

\footnotetext{
${ }^{1}$ At least two triplet scalars are required to break $S U(2)_{D}$ into $Z_{2}$ where unbroken $U(1)$ remains for one triplet case.

${ }^{2}$ In Ref. [3], a similar model is considered where two Dirac fermions are introduced to explain some astrophysical anomalies assuming specific mass relation among DM candidates and hidden gauge bosons.
}

TABLE I. Charge assignment for the fields in $S U(2)_{D}$ dark sector where $\chi$ is Dirac fermion and $\vec{\phi}_{i}(i=1,2)$ are scalars.

\begin{tabular}{lcc}
\hline \hline Fields & $\chi$ & $\vec{\phi}$ \\
\hline \hline$S U(2)_{D}$ & $\mathbf{2}$ & $\mathbf{3}$ \\
\hline \hline$\langle\vec{\phi}\rangle=\left(0,0, \frac{v_{\phi}}{\sqrt{2}}\right)$, & $\left\langle\vec{\phi}^{\prime}\right\rangle=\left(\frac{v_{\phi^{\prime}}}{\sqrt{2}}, 0,0\right)$.
\end{tabular}

When $S U(2)_{D}$ is broken by the VEVs of the triplets, the vacuum is invariant under the transformation defined by $U_{T_{3}} \equiv e^{i 2 \pi T_{3}}$ as $U_{T_{3}}\left\langle\vec{\phi}^{(\prime)}\right\rangle=\left\langle\vec{\phi}^{(\prime)}\right\rangle$, where $T_{3}$ is the diagonal component of $S U(2)_{D}$ generators. Then $\chi$ field transform $U_{T_{3}} \chi=-\chi$ since $T_{3}$ values of $\chi$ 's components are $\pm 1 / 2$. In general, we would obtain even (odd) parity under $U$ transformation for any component of $S U(2)_{D}$ multiplet with integer (half-integer) value of $T_{3}$. Thus $S U(2)_{D}$ gauge symmetry is broken to $Z_{2}$ symmetry when we assume Eq. (2.2). Note that the $Z_{2}$ symmetry will guarantees the stability of DM candidate which is the lightest component with odd $Z_{2}$ parity.

Now we write down the Lagrangian for kinetic terms of the dark sector and the scalar potential:

$$
\begin{aligned}
\mathcal{L}_{D}= & -\frac{1}{4} X_{\mu \nu}^{a} X^{a \mu \nu}+D_{\mu} \vec{\phi} \cdot D^{\mu} \vec{\phi}+D_{\mu} \vec{\phi}^{\prime} \cdot D^{\mu} \vec{\phi}^{\prime} \\
& +\bar{\chi}\left(D_{\mu} \gamma^{\mu}-M_{\chi}\right) \chi \\
V= & \mu_{H}^{2} H^{\dagger} H+\lambda_{H}\left(H^{\dagger} H\right)^{2}+\mu_{1}^{2} \vec{\phi} \cdot \vec{\phi}+\mu_{2}^{2} \vec{\phi}^{\prime} \cdot \vec{\phi}^{\prime}+\lambda_{1}(\vec{\phi} \cdot \vec{\phi})^{2} \\
& +\lambda_{2}\left(\vec{\phi}^{\prime} \cdot \vec{\phi}^{\prime}\right)^{2}+\lambda_{3}\left(\vec{\phi} \cdot \vec{\phi}^{\prime}\right)^{2}+\lambda_{4}(\vec{\phi} \cdot \vec{\phi})\left(\vec{\phi} \cdot \vec{\phi}^{\prime}\right) \\
& +\lambda_{5}\left(\vec{\phi}^{\prime} \cdot \vec{\phi}^{\prime}\right)\left(\vec{\phi} \cdot \vec{\phi}^{\prime}\right)+\lambda_{6}(\vec{\phi} \cdot \vec{\phi})\left(\vec{\phi}^{\prime} \cdot \vec{\phi}^{\prime}\right) \\
& +\lambda_{H \phi}(\vec{\phi} \cdot \vec{\phi})\left(H^{\dagger} H\right)+\lambda_{H \phi^{\prime}}\left(\vec{\phi}^{\prime} \cdot \vec{\phi}^{\prime}\right)\left(H^{\dagger} H\right) \\
& +\frac{y_{\chi \phi}}{2} \bar{\chi}(\vec{\phi} \cdot \vec{\sigma}) \chi+\frac{y_{\chi \phi^{\prime}}}{2} \bar{\chi}\left(\vec{\phi}^{\prime} \cdot \vec{\sigma}\right) \chi
\end{aligned}
$$

where $X_{\mu \nu}^{a}(a=1,2,3)$ is the field strength of $S U(2)_{D}$ gauge field, and $H$ is the SM Higgs doublet field written as

$$
H=\left(\begin{array}{c}
G^{+} \\
\frac{1}{\sqrt{2}}\left(v+\tilde{h}+i G_{Z}\right)
\end{array}\right) .
$$

Here $v_{H}$ is the VEV of the SM Higgs doublet, $H$, and $G^{ \pm}$ and $G_{Z}$ are Nambu-Goldstone(NG) bosons absorbed by $W^{ \pm}$and $Z$ bosons.

\section{A. Scalar sector}

Firstly we briefly discuss our VEV configuration Eq. (2.2) can give global minimum of the potential. In general we choose $\langle\vec{\phi}\rangle=\left(0,0, \frac{v_{\phi}}{\sqrt{2}}\right)$ using freedom of $S U(2)$ rotations. Then the value of scalar potential $V$ will be 
smaller when $\vec{\phi} \cdot \vec{\phi}^{\prime}=0$ for $\lambda_{i}>0(i=1, \ldots, 6)$. Thus $\left\langle\vec{\phi}^{\prime}\right\rangle=$ $\left(v_{\phi_{1}^{\prime}}, v_{\phi_{2}^{\prime}}, 0\right)$ gives global minimum of the potential (for $\left.\mu_{1,2}^{2}<0\right)$. Since the potential minima is symmetric under rotation on $\left(v_{\phi_{1}^{\prime}}, v_{\phi_{2}^{\prime}}\right)$ space we chose $\left\langle\vec{\phi}^{\prime}\right\rangle=\left(\frac{v_{\phi}^{\prime}}{\sqrt{2}}, 0,0\right)$

Here we consider the scalar sector of the model. First we consider conditions to get VEV alignment in Eq. (2.2). From the stationary conditions $\partial V / \partial \phi_{i}=0$ and $\partial V / \partial \phi_{i}^{\prime}=0$, we obtain the following nontrivial conditions (or vanishing tadpole conditions):

$$
\begin{aligned}
\lambda_{1} v_{\phi}^{3}+\frac{1}{2} \lambda_{6} v_{\phi} v_{\phi^{\prime}}^{2}+\frac{1}{2} \lambda_{H \phi} v_{\phi} v_{H}^{2}-\mu_{1}^{2} v_{\phi} & =0, \\
\lambda_{2} v_{\phi^{\prime}}^{3}+\frac{1}{2} \lambda_{6} v_{\phi}^{2} v_{\phi^{\prime}}+\frac{1}{2} v_{\phi} \lambda_{H \phi^{\prime}} v_{H}^{2}-\mu_{2}^{2} v_{\phi^{\prime}} & =0, \\
\lambda_{H} v_{H}^{3}+\frac{1}{2} \lambda_{H \phi} v_{\phi}^{2} v_{H}+\frac{1}{2} \lambda_{H \phi^{\prime}} v_{\phi^{\prime}}^{2} v_{H}-\mu_{H}^{2} v_{H} & =0, \\
\lambda_{4} v_{\phi}^{2}+\lambda_{5} v_{\phi^{\prime}}^{2} & =0 .
\end{aligned}
$$

The mass terms of scalar fields are given by the quadratic terms in the scalar fields in the Lagrangian:

$$
\begin{aligned}
-\mathcal{L}_{M_{S}}= & \frac{1}{4} \lambda_{3} v_{\phi^{\prime}}^{2} \phi_{1}^{2}+\frac{1}{4} \lambda_{4} v_{\phi} v_{\phi^{\prime}} \phi_{1} \phi_{3}-\frac{1}{2} \lambda_{4} v_{\phi}^{2} \phi_{1} \phi_{1}^{\prime} \\
& +\frac{1}{2} \lambda_{3} v_{\phi} v_{\phi^{\prime}} \phi_{1} \phi_{3}^{\prime}+\lambda_{1} v_{\phi}^{2} \phi_{3}^{2}+\lambda_{6} v_{\phi} v_{\phi^{\prime}} \phi_{3} \phi_{1}^{\prime} \\
& +\frac{1}{2} \lambda_{4} v_{\phi}^{2} \phi_{3} \phi_{3}^{\prime}+\lambda_{2} v_{\phi^{\prime}}^{2} \phi_{1}^{\prime 2}-\frac{1}{2} \lambda_{4} \frac{v_{\phi}^{3}}{v_{\phi}^{\prime}} \phi_{1}^{\prime} \phi_{3}^{\prime}+\frac{1}{4} \lambda_{3} v_{\phi}^{2} \phi_{3}^{\prime 2} \\
& +\lambda_{H} v_{H}^{2} \tilde{h}^{2}+\lambda_{H \phi} v_{\phi} v_{H} \phi_{3} \tilde{h}+\lambda_{H \phi^{\prime}} v_{\phi^{\prime}} v_{H} \phi_{1}^{\prime} \tilde{h},
\end{aligned}
$$

where we used the last equation of Eq. (2.6) to substitute $\lambda_{5}$. Notice that mass terms associated with $\phi_{2}$ and $\phi_{2}^{\prime}$ are absent and they are identified as Nambu-Goldstone(NG) bosons which are absorbed by the two massive gauge bosons in the dark sector.

From now on, we shall assume $\lambda_{4} \ll 1$ to simplify the scalar mass terms. Then Eq. (2.7) becomes

$$
\begin{aligned}
-\mathcal{L}_{M_{S}} \simeq & \frac{1}{4} \lambda_{3} v_{\phi^{\prime}}^{2} \phi_{1}^{2}+\frac{1}{2} \lambda_{3} v_{\phi} v_{\phi^{\prime}} \phi_{1} \phi_{3}^{\prime}+\lambda_{1} v_{\phi}^{2} \phi_{3}^{2} \\
& +\lambda_{6} v_{\phi} v_{\phi^{\prime}} \phi_{3} \phi_{1}^{\prime}+\lambda_{2} v_{\phi^{\prime}}^{2} \phi_{1}^{\prime 2}+\frac{1}{4} \lambda_{3} v_{\phi}^{2} \phi_{3}^{\prime 2}+\lambda_{H} v_{H}^{2} \tilde{h}^{2} \\
& +\lambda_{H \phi} v_{\phi} v_{H} \phi_{3} h+\lambda_{H \phi^{\prime}} v_{\phi^{\prime}} v_{H} \phi_{1}^{\prime} h
\end{aligned}
$$

ignoring terms with the $\lambda_{4}$ coupling. The terms for $\phi_{1}$ and $\phi_{3}^{\prime}$ can be organized as

$$
\frac{1}{4} \lambda_{3}\left(v_{\phi}^{2}+v_{\phi^{\prime}}^{2}\right)\left(\cos \alpha \phi_{1}+\sin \alpha \phi_{3}^{\prime}\right)^{2},
$$

where $\sin \alpha(\cos \alpha)=v_{\phi^{\prime}}\left(v_{\phi}\right) / \sqrt{v_{\phi}^{2}+v_{\phi^{\prime}}^{2}}$. We then find that the mass eigenstate $\left(\cos \alpha \phi_{1}+\sin \alpha \phi_{3}^{\prime}\right)$ has the mass eigenvalue $\lambda_{3}\left(v_{\phi}^{2}+v_{\phi^{\prime}}^{2}\right) / 2$, whereas its orthogonal state $\left(-\sin \alpha \phi_{1}+\cos \alpha \phi_{3}^{\prime}\right)$ corresponds to the NG boson absorbed by $S U(2)_{D}$ dark gauge boson.

Finally, the mass matrix for $\left(h, \phi_{3}, \phi_{1}^{\prime}\right)$ is given by

$$
\begin{aligned}
-\mathcal{L}_{M_{S}} \supset & \frac{1}{2}\left(\begin{array}{c}
h \\
\phi_{3} \\
\phi_{1}^{\prime}
\end{array}\right)^{T}\left(\begin{array}{ccc}
2 \lambda_{H} v_{H}^{2} & \lambda_{H \phi} v_{\phi} v_{H} & \lambda_{H \phi^{\prime}} v_{\phi^{\prime}} v_{H} \\
\lambda_{H \phi} v_{\phi} v_{H} & 2 \lambda_{1} v_{\phi}^{2} & \lambda_{6} v_{\phi} v_{\phi^{\prime}} \\
\lambda_{H \phi^{\prime}} v_{\phi^{\prime}} v_{H} & \lambda_{6} v_{\phi} v_{\phi^{\prime}} & 2 \lambda_{2} v_{\phi^{\prime}}^{2}
\end{array}\right) \\
& \times\left(\begin{array}{c}
h \\
\phi_{3} \\
\phi_{1}^{\prime}
\end{array}\right) .
\end{aligned}
$$

Thus $\phi_{3}$ and $\phi_{1}^{\prime}$ can mix with the SM Higgs field and interact with SM particle via mixing effects. In our phenomenological analysis, we discuss the following two simplified cases. Scenario (1): $\lambda_{H \phi^{\prime}}, \lambda_{6} \rightarrow 0$ In this case, $\tilde{h}$ and $\phi_{3}$ mix while $\phi_{1}^{\prime}$ is almost the mass eigenstate without mixing. Then squared mass terms for $\left\{\tilde{h}, \phi_{3}\right\}$ are given by

$-\mathcal{L} \supset \frac{1}{2}\left(\begin{array}{c}\tilde{h} \\ \phi_{3}\end{array}\right)^{T}\left(\begin{array}{cc}2 \lambda_{H} v_{H}^{2} & \lambda_{H \phi} v_{\phi} v_{H} \\ \lambda_{H \phi} v_{\phi} v_{H} & 2 \lambda_{1} v_{\phi}^{2}\end{array}\right)\left(\begin{array}{c}\tilde{h} \\ \phi_{3}\end{array}\right)$.

This squared mass matrix can be diagonalized by an orthogonal matrix, and the resulting mass eigenvalues are given by

$m_{h, \Phi_{1}}^{2}=\lambda_{H} v_{H}^{2}+\lambda_{1} v_{\phi}^{2} \pm \sqrt{\left(\lambda_{H} v_{H}^{2}-\lambda_{1} v_{\phi}^{2}\right)^{2}+\lambda_{H \phi}^{2} v_{\phi}^{2} v_{H}^{2}}$.

The relevant mass eigenstates $h$ and $\Phi_{1}$ are also given by

$$
\begin{aligned}
\left(\begin{array}{c}
h \\
\Phi_{1}
\end{array}\right) & =\left(\begin{array}{cc}
\cos \alpha & \sin \alpha \\
-\sin \alpha & \cos \alpha
\end{array}\right)\left(\begin{array}{c}
\tilde{h} \\
\phi_{3}
\end{array}\right), \\
\tan 2 \alpha & =\frac{\lambda_{H \varphi} v_{\phi} v_{H}}{\lambda_{H} v_{H}^{2}-\lambda_{1} v_{\phi}^{2}},
\end{aligned}
$$

where $\alpha$ is the mixing angle, and $h$ is identified as the SMlike Higgs boson. Also we rewrite $\phi_{1}^{\prime}$ as an approximate mass eigenstate such that

$$
\Phi_{2} \simeq \phi_{1}^{\prime}, \quad m_{\Phi_{2}}^{2} \simeq 2 \lambda_{2} v_{\phi^{\prime}}^{2}
$$

The scalar mixing is constrained by Higgs precision measurements as $\sin \alpha \lesssim 0.3$ when the SM Higgs does not decay into particles in the dark sector $[16,17]$ We investigate the bound for $\sin \alpha$ when the SM Higgs decays into dark gauge bosons below. Scenario (2): $\lambda_{H \phi}, \lambda_{6} \rightarrow 0$ In this case, we obtain mass eigenvalues and eigenstates by replacement $\lambda_{H \phi} \rightarrow \lambda_{H \phi^{\prime}}, v_{\phi} \rightarrow v_{\phi^{\prime}}, \lambda_{1} \leftrightarrow \lambda_{2}$ and $\phi_{3} \leftrightarrow \phi_{1}^{\prime}$ for case (1). Then they are given by 


$$
\begin{gathered}
m_{h, \Phi_{1}^{\prime}}^{2}=\lambda_{H} v_{H}^{2}+\lambda_{2} v_{\phi^{\prime}}^{2} \pm \sqrt{\left(\lambda_{H} v_{H}^{2}-\lambda_{2} v_{\phi^{\prime}}^{2}\right.} \\
\left(\begin{array}{c}
h \\
\Phi_{1}^{\prime}
\end{array}\right)=\left(\begin{array}{cc}
\cos \alpha^{\prime} & \sin \alpha^{\prime} \\
-\sin \alpha^{\prime} & \cos \alpha^{\prime}
\end{array}\right)\left(\begin{array}{c}
\tilde{h} \\
\phi_{1}^{\prime}
\end{array}\right), \\
\tan 2 \alpha^{\prime}=\frac{\lambda_{H \varphi^{\prime}} v_{\phi^{\prime}} v_{H}}{\lambda_{H} v_{H}^{2}-\lambda_{2} v_{\phi^{\prime}}^{2}},
\end{gathered}
$$

where $\alpha^{\prime}$ is the mixing angle, and $h$ is again identified as the SM-like Higgs boson. Also we rewrite $\phi_{3}$ as approximate mass eigenstate such that

$$
\Phi_{2}^{\prime} \simeq \phi_{3}, \quad m_{\Phi_{2}^{\prime}}^{2} \simeq 2 \lambda_{1} v_{\phi}^{2}
$$

\section{B. Gauge sector}

The dark and the SM sectors can interact through terms in the potential associated with the SM Higgs in Eq. (2.4) that is called the Higgs portal. In addition the dark gauge sector and the SM gauge sector can be concocted via kinetic mixings between $S U(2)_{D}$ and $U(1)_{Y}$ after $S U(2)_{D}$ gauge symmetry breaking by nonzero VEVs of $\vec{\phi}$ and $\vec{\phi}^{\prime}$ [18]. The relevant terms for these kinetic mixings are two dim-5 operators:

$$
\mathcal{L}_{X B}=\frac{C_{\phi}}{\Lambda} X_{\mu \nu}^{a} B^{\mu \nu} \phi^{a}+\frac{C_{\phi^{\prime}}}{\Lambda} X_{\mu \nu}^{a} B^{\mu \nu} \phi^{\prime a},
$$

where $\Lambda$ indicate the cut off scale and $B^{\mu \nu}$ is the gauge field strength for $U(1)_{Y}{ }^{3}$ After $\phi$ and $\phi^{\prime}$ developing VEVs, we obtain the following kinetic mixing terms:

$$
\mathcal{L}_{\mathrm{KM}}=-\frac{1}{2} \sin \delta_{1} X_{\mu \nu}^{1} B^{\mu \nu}-\frac{1}{2} \sin \delta_{3} X_{\mu \nu}^{3} B^{\mu \nu},
$$

where we defined $\sin \delta_{1} \equiv \sqrt{2} C_{\phi^{\prime}} v_{\phi} / \Lambda$ and $\sin \delta_{3} \equiv$ $\sqrt{2} C_{\phi} v_{\phi^{\prime}} / \Lambda$ as new kinetic mixing parameters.

The kinetic terms for $X_{\mu}^{1,3}$ and $B_{\mu}$ can be diagonalized by the following transformations:

$B_{\mu}=\tilde{B}_{\mu}-\tan \delta_{1} \tilde{X}_{\mu}^{1}-\frac{1}{\cos \delta}\left(\tan \delta_{3}-\tan \delta_{1} \sin \delta\right) \tilde{X}_{\mu}^{3}$,

\footnotetext{
${ }^{3}$ The effective operator can be obtained when there is a field with nonzero $U(1)_{B}$ charge and nontrivial $S U(2)_{X}$ representation. For example, it is possible to obtain the effective operator at one loop level when we introduce extra fermion $E$ whose representation is $2(-1)$ under $S U(2)_{X}\left(U(1)_{B}\right)$. Such one-loop diagrams are obtained from interactions: $g_{X} \bar{E} \gamma^{\mu} X_{\mu} E-$ $g_{B} \bar{E} \gamma^{\mu} B_{\mu} E+y \bar{E} \phi_{a}^{(\prime)} \sigma^{a} / 2 E$ where $\sigma^{a}$ is the Pauli matrix acting on $S U(2)_{X}$ representation space.
}

$$
\begin{gathered}
X_{\mu}^{1}=\frac{1}{\cos \delta_{1}} \tilde{X}_{\mu}^{1}-\frac{\tan \delta}{\cos \delta_{1}} X_{\mu}^{3}, \\
X_{\mu}^{3}=\frac{1}{\cos \delta_{3} \cos \delta} \tilde{X}_{\mu}^{3},
\end{gathered}
$$

where $\delta$ is defined as $\sin \delta \equiv-\tan \delta_{1} \tan \delta_{3}$. In our analysis, we take a limit of $\delta_{1} \ll 1$ and $\delta_{3} \ll 1$ and gauge fields are approximately written by

$$
B \simeq \tilde{B}-\delta_{1} X_{\mu}^{1}-\delta_{3} X_{\mu}^{3}, \quad X_{\mu}^{1} \simeq \tilde{X}_{\mu}^{1}, \quad X_{\mu}^{3} \simeq \tilde{X}_{\mu}^{3} .
$$

Then we denote dark gauge bosons associated with $X_{\mu}^{1,2,3}$ field as $X_{1,2,3}$ henceforth. Note that mixing with $Z$ boson is suppressed unless dark gauge boson and the SM $Z$ boson masses are not close enough. In our analysis, we assume a dark gauge boson mainly mixes with photon field.

After two triplet scalar fields develop nonzero VEVs, $S U(2)_{D}$ gauge bosons obtain masses from kinetic term such that

$\mathcal{L}_{M}=g_{D}^{2} v_{\phi}^{2} X_{\mu}^{1} X^{1 \mu}+g_{D}^{2}\left(v_{\phi}^{2}+v_{\phi^{\prime}}^{2}\right) X_{\mu}^{2} X^{2 \mu}+g_{D}^{2} v_{\phi^{\prime}}^{2} X_{\mu}^{3} X^{3 \mu}$.

Here we have ignored kinetic mixing effects since it is negligibly small in our scenario. We thus find the masses of dark gauge bosons to be

$m_{X_{1}}=\sqrt{2} g_{D} v_{\phi}, \quad m_{X_{2}}=g_{D} \sqrt{2\left(v_{\phi}^{2}+v_{\phi^{\prime}}^{2}\right)}$,

$m_{X_{3}}=\sqrt{2} g_{D} v_{\phi^{\prime}}$.

Note that the $X_{2}$ is always the heaviest one. In addition, three-point interactions among scalar and gauge bosons are given by

$\mathcal{L} \supset g_{D}^{2} v_{\phi} \phi_{3}\left(X_{\mu}^{1} X^{1 \mu}+X_{\mu}^{2} X^{2 \mu}\right)+g_{D}^{2} v_{\phi^{\prime}} \phi_{1}^{\prime}\left(X_{\mu}^{2} X^{2 \mu}+X_{\mu}^{3} X^{3 \mu}\right)$,

where $\phi_{3}$ and $\phi_{1}^{\prime}$ can be written as mass eigenstates using Eqs. (2.13) and (2.14) for the case (1) and using Eqs (2.16) and (2.17) for the case (2) described in a previous subsection.

Finally interactions among dark gauge fields are also written $\rightarrow$ given by

$\mathcal{L} \supset-g_{D} \epsilon^{a b c} \partial_{\mu} X_{\nu}^{a} X^{b \mu} X^{c \nu}-\frac{1}{4} g_{D}^{2} \epsilon^{a b c} \epsilon^{a d e} X_{\mu}^{b} X_{\nu}^{c} X^{d \mu} X^{e \nu}$,

where $\epsilon^{a b c}$ is the structure constants of $S U(2)_{D}$ and $a=1$, 2,3 . The heaviest gauge boson $X_{2}$ would decay into $X_{1} X_{3}$ through the three point gauge interaction, where the $X_{1}$ and/ or $X_{3}$ transition will be off-shell due to the mass relation 
among dark gauge bosons and both of them will eventually decay into the SM particles through kinetic mixings, Eq. (2.19).

\section{Fermions in the dark sector}

The mass terms of $S U(2)_{D}$ doublet fermion are given by

$$
\begin{aligned}
-\mathcal{L} \supset & M_{\chi}\left(\bar{\chi}_{1} \chi_{1}+\bar{\chi}_{2} \chi_{2}\right)+\frac{y_{\chi \phi} v_{\phi}}{2}\left(\bar{\chi}_{1} \chi_{1}-\bar{\chi}_{2} \chi_{2}\right) \\
& +\frac{y_{\chi \phi^{\prime}} v_{\phi^{\prime}}}{2}\left(\bar{\chi}_{1} \chi_{2}+\bar{\chi}_{2} \chi_{1}\right) \\
\equiv & M_{11} \bar{\chi}_{1} \bar{\chi}_{1}+M_{12}\left(\bar{\chi}_{1} \chi_{2}+\bar{\chi}_{2} \chi_{1}\right)+M_{22} \bar{\chi}_{2} \chi_{2},
\end{aligned}
$$

where we assumed all coefficients are real. The mass splitting and the mass mixings between $\chi_{1}$ and $\chi_{2}$ are induced by the $y_{\chi \phi}$ and $y_{\chi \phi^{\prime}}$ respectively, in the last line of Eq. (2.4). The mass eigenvalues and eigenstates are obtained in a straightforward manner as

$$
\begin{aligned}
& m_{\chi_{l}, \chi_{h}}=\frac{1}{2}\left(M_{11}+M_{22}\right) \pm \frac{1}{2} \sqrt{\left(M_{11}-M_{22}\right)^{2}+4 M_{12}^{2}} \\
& \left(\begin{array}{l}
\chi_{1} \\
\chi_{2}
\end{array}\right)=\left(\begin{array}{cc}
\cos \theta_{\chi} & -\sin \theta_{\chi} \\
\sin \theta_{\chi} & \cos \theta_{\chi}
\end{array}\right)\left(\begin{array}{l}
\chi_{l} \\
\chi_{h}
\end{array}\right)
\end{aligned}
$$

where $m_{\chi_{l}}<m_{\chi_{h}}$ by definition. The mixing angle $\theta_{\chi}$ is given by

$$
\tan 2 \theta_{\chi}=\frac{2 M_{12}}{M_{11}-M_{22}}=\frac{y_{\chi \phi^{\prime}} v_{\phi^{\prime}}}{y_{\chi \phi} v_{\phi}}
$$

Furthermore we obtain interactions among scalar fields and mass eigenstates of dark fermions such that

$$
\begin{aligned}
\mathcal{L} & \supset \frac{y_{\chi \phi}}{2}\left[\phi_{3}\left(\cos 2 \theta_{\chi} \bar{\chi}_{l} \chi_{l}-\cos 2 \theta_{\chi} \bar{\chi}_{h} \chi_{h}-\sin 2 \theta_{\chi}\left(\bar{\chi}_{l} \chi_{h}+\bar{\chi}_{h} \chi_{l}\right)\right)\right. \\
& \left.+\phi_{1}\left(\sin 2 \theta_{\chi} \bar{\chi}_{l} \chi_{l}-\sin 2 \theta_{\chi} \bar{\chi}_{h} \chi_{h}+\cos 2 \theta_{\chi}\left(\bar{\chi}_{l} \chi_{h}+\bar{\chi}_{h} \chi_{l}\right)\right)\right] \\
& +\frac{y_{\chi \phi^{\prime}}}{2}\left[\phi _ { 3 } ^ { \prime } \left(\cos 2 \theta_{\chi} \bar{\chi}_{l} \chi_{h}-\cos 2 \theta_{\chi} \bar{\chi}_{h} \chi_{h}\right.\right. \\
& \left.-\sin 2 \theta_{\chi}\left(\bar{\chi}_{l} \chi_{h}+\bar{\chi}_{h} \chi_{l}\right)\right) \\
& \left.+\phi_{1}^{\prime}\left(\sin 2 \theta_{\chi} \bar{\chi}_{l} \chi_{l}-\sin 2 \theta_{\chi} \bar{\chi}_{h} \chi_{h}+\cos 2 \theta_{\chi}\left(\bar{\chi}_{l} \chi_{h}+\bar{\chi}_{h} \chi_{l}\right)\right)\right]
\end{aligned}
$$

where $\phi_{1,3}$ and $\phi_{1,3}^{\prime}$ are substituted to mass eigenstates as discussed in previous subsection.

\section{Topological $Z_{2}$ string}

In our DM model, the original non-Abelian gauge symmetry $G \equiv S U(2)_{D}$ is spontaneously broken down to its subgroup $H=Z_{2}=\{1,-1\}$ which is disconnected. Then the vacuum manifold of the model is given by $\mathcal{M}=$ $G / H$ so that the first homotopy group of $\mathcal{M}$ is $\pi_{1}(G / H)=$ $\pi_{0}(H)=H=Z_{2}[19]$. Therefore in the particle spectra of this model, there will be $Z_{2}$ string which is a topological object. One $Z_{2}$ vortex is topologically nontrivial, but two of them can be deformed smoothly into the vacuum, thereby being topologically trivial. These $Z_{2}$ string can contribute to the dark matter of the current Universe to some extent, but detailed study of this issue is beyond the scope of this paper. In the following, we shall simply ignore topological $Z_{2}$ strings assuming their contribution to the Universe is negligible.

\section{DARK MATTER}

In this section, we discuss DM phenomenology in our model including DM relic density. In our scenario, DM is the lightest dark fermion $\chi_{l}$ which is stabilized by the remnant dark $Z_{2}$ symmetry after dark gauge symmetry breaking. First we require that Higgs portal interactions of DM are suppressed in order to avoid severe constraints from DM direct detection experiments. For the scenario (1) of Higgs mixing, we prefer large mixing of dark fermions, $\theta_{\chi} \sim \pi / 4$, since DM couples with Higgs via $\phi_{3}$. On the other hand, for the scenario (2), we prefer small mixing, $\left|\theta_{\chi}\right| \ll 1$, since DM couples with Higgs via $\phi_{1}^{\prime}$. Then relic density of DM is determined by gauge interactions in dark sector in our scenarios where we assume dark scalars are heavier than DM.

Then, the relevant interaction terms are

$$
\begin{aligned}
\mathcal{L} \supset & \frac{g_{D}}{2}\left(\cos 2 \theta_{\chi} \bar{\chi}_{l} \gamma^{\mu} \chi_{l}-\sin 2 \theta_{\chi} \bar{\chi}_{l} \gamma^{\mu} \chi_{h}-\sin 2 \theta_{\chi} \bar{\chi}_{h} \gamma^{\mu} \chi_{l}\right. \\
& \left.-\cos 2 \theta_{\chi} \bar{\chi}_{h} \gamma^{\mu} \chi_{h}\right) X_{\mu}^{3} \\
& +\frac{g_{D}}{2}\left(\sin 2 \theta_{\chi} \bar{\chi}_{l} \gamma^{\mu} \chi_{l}+\cos 2 \theta_{\chi} \bar{\chi}_{l} \gamma^{\mu} \chi_{h}\right. \\
& \left.+\cos 2 \theta_{\chi} \bar{\chi}_{h} \gamma^{\mu} \chi_{l}-\sin 2 \theta_{\chi} \bar{\chi}_{h} \gamma^{\mu} \chi_{h}\right) X_{\mu}^{1} \\
& +i \frac{g_{D}}{2}\left(\bar{\chi}_{h} \gamma^{\mu} \chi_{l}-\bar{\chi}_{l} \gamma^{\mu} \chi_{h}\right) X_{\mu}^{2}+e c_{W} \delta_{1} X_{\mu}^{1} J_{\mathrm{EM}}^{\mu} \\
& +e c_{W} \delta_{3} X_{\mu}^{3} J_{\mathrm{EM}}^{\mu}, \\
& J_{\mathrm{EM}}^{\mu}=\sum_{f_{\mathrm{SM}}} Q_{f_{\mathrm{SM}}} \bar{f}_{\mathrm{SM}} \gamma^{\mu} f_{\mathrm{SM}}
\end{aligned}
$$

where $J_{\mathrm{EM}}^{\mu}$ is electromagnetic current and $Q_{f_{\mathrm{SM}}}$ is the electric charge of the SM fermions $f_{\mathrm{SM}}$. Then we implement these interactions in MICROMEGAS 4.3.5 [20] to estimate relic density where all annihilation and coannihilation processes induced by these interactions are included in the numerical analysis.

In our analysis we take the dark fermion mixing angle for each scenario as

$$
\begin{aligned}
& \theta_{\chi}=\frac{\pi}{4} \text { for the scenario (1), } \\
& \theta_{\chi}=0 \text { for the scenario (2), }
\end{aligned}
$$



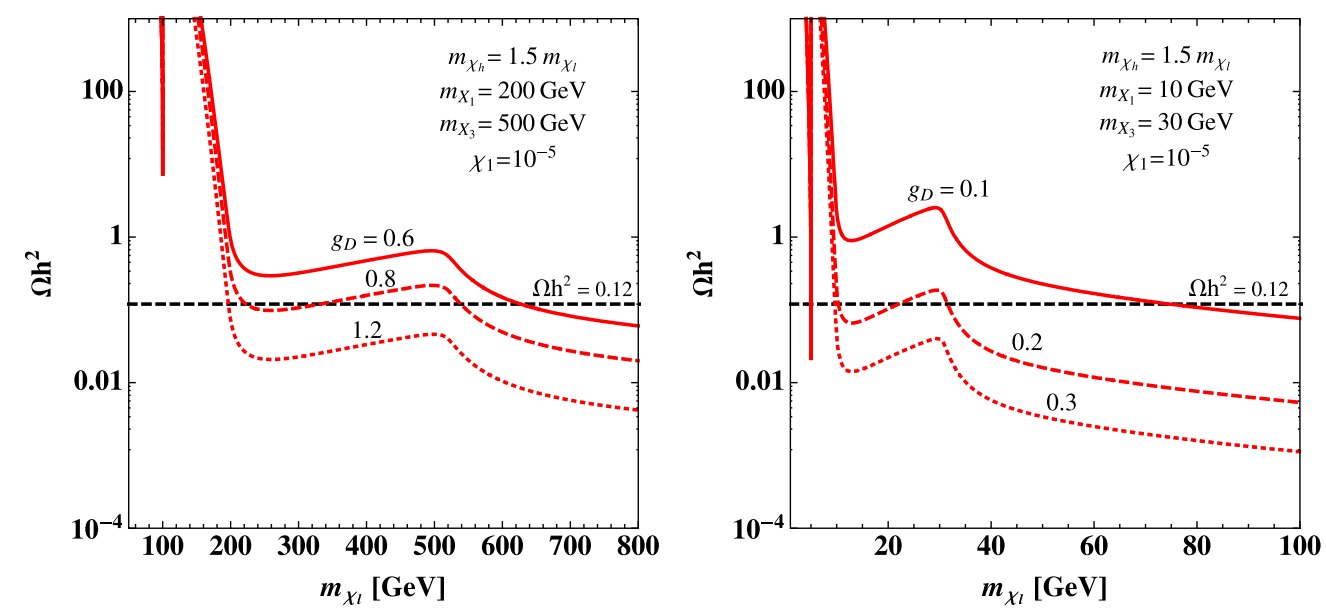

FIG. 1. Relic density as a function of DM mass where relevant parameters are fixed as indicated on the plots.

in order to avoid direct detection constraint. For illustration, we consider two benchmark cases of dark gauge boson masses for each scenario;

$$
\begin{aligned}
& \text { Scenario (1): } m_{X_{1}}<m_{X_{3}}, \\
& \text { Scenario (2): } m_{X_{1}}>m_{X_{3}},
\end{aligned}
$$

and $m_{X_{2}}=\sqrt{m_{X_{1}}^{2}+m_{X_{3}}^{2}}$. Also we require interaction between DM and the lightest dark gauge boson not to be suppressed by the dark fermion mixing effect. In the following, we shall focus on the scenario (1) since we just obtain similar results by replacing the role of $X_{1}$ and $X_{3}$ for the scenario (2).

In addition, we take into account $D M$-nucleon scattering via $Z^{\prime}$ boson exchanging process. The cross section for this process is calculated in nonrelativistic limit as

$$
\sigma \simeq \frac{\delta_{1}^{2} g_{D}^{4}}{2 \pi} \frac{1}{m_{X_{1}}^{4}}\left(\frac{m_{\chi_{l}} m_{p}}{m_{\chi_{l}}+m_{p}}\right)^{2}
$$

where $m_{p}$ indicates the proton mass. Since the $Z^{\prime}$-SM fermion interaction is proportional to electromagnetic current, the DM scattering with neutron is suppressed. For $m_{\chi_{l}}=\mathcal{O}(100) \mathrm{GeV}$, we obtain

$$
\sigma \sim 6 \delta_{1}^{2} g_{D}^{4}\left(\frac{100 \mathrm{GeV}}{m_{X_{1}}}\right)^{4} \times 10^{-37} \mathrm{~cm}^{2} .
$$

We then assume $\delta_{1} \lesssim 10^{-5}$ to avoid direct detection constraints such as XENON1T [21] and PandaX-II [22] which provide upper limit of $\sim 10^{-46} \mathrm{~cm}^{2}$ for DM mass of $\sim 100 \mathrm{GeV}$.

In Fig. 1, we show thermal relic density of DM, adopting dark gauge boson masses $\left\{m_{X_{1}}, m_{X_{3}}\right\}$ as $\{200,500\} \mathrm{GeV}$ and $\{10,30\} \mathrm{GeV}$ as reference values, $m_{\chi_{h}}=1.5 m_{\chi_{l}}$, $\delta_{1,3}=10^{-5}$, and some relevant values of gauge coupling
$g_{D}$. We find that relic density is decreased when $\chi_{l} \bar{\chi}_{l} \rightarrow$ $X_{1} X_{1}$ and $\chi_{1} \bar{\chi}_{l} \rightarrow X_{2} X_{2}$ processes are kinematically allowed. Then larger gauge coupling is required for larger $m_{X_{1}}$ mass to accommodate with observed thermal relic density of DM. We can also explain relic density around resonance $2 m_{X_{\chi_{1}}} \sim m_{X_{1}}$ when $X_{1}$ mass is relatively light while the relic density tends to be larger than observed one for heavier dark gauge boson case due to small kinetic mixing parameter.

Next we scan free parameters fixing $\delta_{1,3}=10^{-5}$ to avoid direct detection constraint. The two region for masses of $\chi_{l, h}$ and $X_{1,3}$ are considered in the range of

$$
\begin{aligned}
& \text { Region I: } m_{\chi_{l}} \in[1,50] \mathrm{GeV}, \quad m_{\chi_{h}}=1.5 m_{\chi_{l}} \text {, } \\
& m_{X_{1}} \in[5,20] \mathrm{GeV}, \quad m_{X_{3}}=1.5 m_{X_{1}}, \\
& g_{D} \supset[0.01,1],
\end{aligned}
$$

Region II: $m_{\chi_{l}} \in[50,1000] \mathrm{GeV}, \quad m_{\chi_{h}}=1.5 m_{\chi_{l}}$,

$$
\begin{aligned}
m_{X_{1}} & \in[150,1000] \mathrm{GeV}, \quad m_{X_{3}}=1.5 m_{X_{1}}, \\
g_{D} & \supset[0.05,2],
\end{aligned}
$$

where masses of $\chi_{h}$ and $X_{3}$ are respectively determined by those of $\chi_{l}$ and $X_{1}$ for simplicity. We then search for the parameter region which provide observed DM thermal relic density approximately in the range of $0.11<\Omega h^{2}<0.13$. In the left and right panels of Fig. 2, we show allowed parameter region on $\left\{m_{\mathrm{DM}}, m_{X_{1}}\right\}$ plane for the region I and II where color gradient indicates values of $g_{D}$. We find large allowed region when $\chi_{l} \bar{\chi}_{l} \rightarrow X_{1} X_{1}\left(X_{2} X_{2}\right)$ processes are kinematically allowed. On the other hand, for $m_{X_{1}}>m_{\chi l}$, we need some fine tuning around $m_{X_{1}} \simeq 2 m_{\chi_{l}}$ to obtain resonant enhancement of annihilation cross section. 

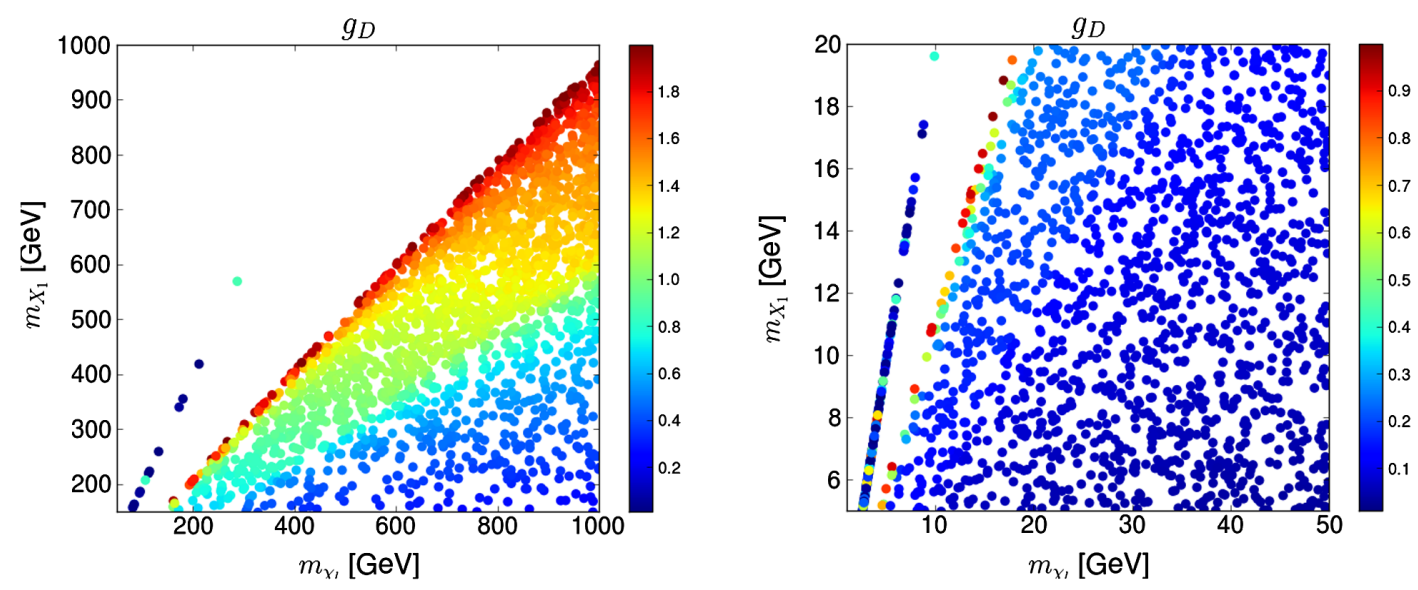

FIG. 2. Parameter region satisfying relic density of DM.

\section{IMPLICATIONS IN COLLIDER PHYSICS}

Here we discuss collider physics in the model focusing on extra scalar boson production at the LHC via Higgs mixing. Then extra scalar boson decays into dark gauge boson, dark fermion or SM particles where the branching ratio (BR) depends on parameters in the model. We then discuss possible signals of the model at the LHC.

\section{A. Constraint from the SM Higgs boson decay}

First we discuss constraints from the SM Higgs decay process, $h \rightarrow X_{1,2,3} X_{1,2,3} \rightarrow \ell^{+} \ell^{-} \ell^{+} \ell^{-}$where $\ell$ denotes electron or muon. This multilepton decay channel is strongly constrained by the search for Higgs boson decaying into extra gauge boson which can decay into charged leptons [23] because of little background. The decay $h \rightarrow X_{1,2,3} X_{1,2,3}$ is induced via scalar mixing between the dark sector and the SM Higgs sector. For the scenario (1), we obtain the decay widths as

$$
\begin{gathered}
\Gamma_{h \rightarrow X_{1} X_{1}}=\frac{g_{D}{ }^{4} \cos ^{2} \alpha}{8 \pi} \frac{v_{\phi}^{2}}{m_{h}} \sqrt{1-\frac{4 m_{X_{1}}^{2}}{m_{h}^{2}}}\left[2+\frac{m_{h}^{4}}{4 m_{X_{1}}^{4}}\left(1-\frac{2 m_{X_{1}}^{2}}{m_{h}^{2}}\right)^{2}\right], \\
\Gamma_{h \rightarrow X_{2} X_{2}}=\frac{g_{D}{ }^{4} \cos ^{2} \alpha}{8 \pi} \frac{v_{\phi}^{2}}{m_{h}} \sqrt{1-\frac{4 m_{X_{2}}^{2}}{m_{h}^{2}}}\left[2+\frac{m_{h}^{4}}{4 m_{X_{2}}^{4}}\left(1-\frac{2 m_{X_{2}}^{2}}{m_{h}^{2}}\right)^{2}\right] .
\end{gathered}
$$

For the scenario (2), we also obtain the decay widths as

$$
\begin{gathered}
\Gamma_{h \rightarrow X_{3} X_{3}}=\frac{g_{D}{ }^{4} \cos ^{2} \alpha^{\prime}}{8 \pi} \frac{v_{\phi^{\prime}}^{2}}{m_{h}} \sqrt{1-\frac{4 m_{X_{3}}^{2}}{m_{h}^{2}}}\left[2+\frac{m_{h}^{4}}{4 m_{X_{3}}^{4}}\left(1-\frac{2 m_{X_{3}}^{2}}{m_{h}^{2}}\right)^{2}\right], \\
\Gamma_{h \rightarrow X_{2} X_{2}}=\frac{g_{D}{ }^{4} \cos ^{2} \alpha^{\prime}}{8 \pi} \frac{v_{\phi^{\prime}}^{2}}{m_{h}} \sqrt{1-\frac{4 m_{X_{2}}^{2}}{m_{h}^{2}}}\left[2+\frac{m_{h}^{4}}{4 m_{X_{2}}^{4}}\left(1-\frac{2 m_{X_{2}}^{2}}{m_{h}^{2}}\right)^{2}\right] .
\end{gathered}
$$

In Fig. 3, we show branching ratio (BR) for the process $h \rightarrow X_{1} X_{1} \rightarrow \ell^{+} \ell^{-} \ell^{+} \ell^{-}$in the scenario (1) where we consider $2 m_{X_{1}}<m_{h}$ and $2 m_{X_{2,3}}<m_{h}$ for simplicity; for the scenario (2) we obtain the same result replacing the role of $X_{1}$ and $X_{3}$. We also show the upper limit on the BR as a dashed horizontal line. It is then found that the scalar mixing angle and/or the gauge coupling $g_{D}$ should be suppressed when the SM Higgs can decay into dark gauge boson decaying into charged leptons.

\section{B. Scalar boson production}

Here we discuss $\Phi_{1}\left(\Phi_{1}^{\prime}\right)$ production processes at the LHC. The scalar boson can be produced by gluon fusion process $g g \rightarrow \Phi_{1}\left(\Phi_{1}^{\prime}\right)$ through the mixing with the SM 

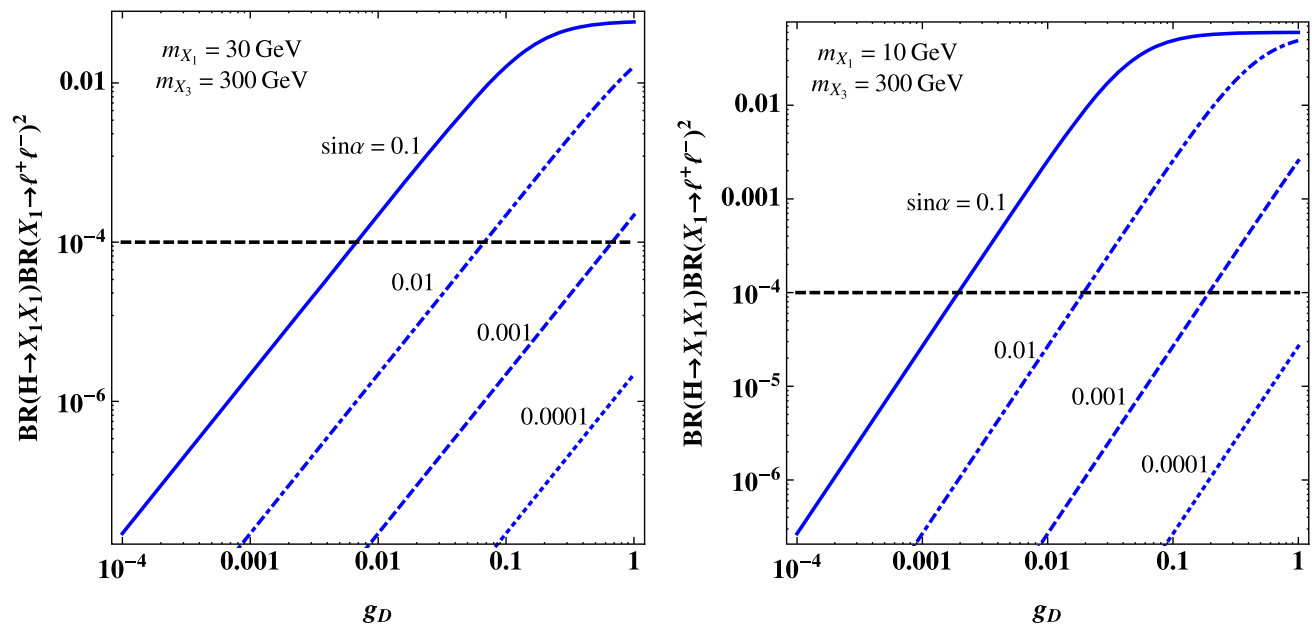

FIG. 3. Branching ratio for $h \rightarrow X_{1} X_{1} \rightarrow \ell^{+} \ell^{-} \ell^{+} \ell^{-}$as a function of $S U(2)_{D}$ gauge coupling $g_{D}$.

Higgs boson parametrized by mixing angle $\alpha\left(\alpha^{\prime}\right)$. The relevant effective interaction for the gluon fusion is written as [24]

$$
\mathcal{L}_{\phi g g}=\frac{\alpha_{s}}{16 \pi} \frac{\sin \alpha\left[\alpha^{\prime}\right]}{v} A_{1 / 2}\left(\tau_{t}\right) \phi G_{\mu \nu}^{a} G^{a \mu \nu},
$$

where $G_{\mu \nu}^{a}$ is the field strength for gluon and $A_{1 / 2}\left(\tau_{t}\right)=$ $-\frac{1}{4}\left[\ln \left[\left(1+\sqrt{\tau_{t}}\right) /\left(1-\sqrt{\tau_{t}}\right)\right]-i \pi\right]^{2} \quad$ with $\tau_{t}=4 m_{t}^{2} / m_{\phi}^{2}$. We obtain this effective interaction from $\bar{t} t \Phi_{1}\left(\Phi_{1}^{\prime}\right)$ coupling via the mixing effect where we take into account only top Yukawa coupling since the other contributions are subdominant. Here we estimate the gluon fusion cross section with CalcHEP [25] by use of the CTEQ6 parton distribution functions (PDFs) [26], implementing the relevant interaction. In addition we apply K-factor $K_{g g}=1.6$ for gluon fusion process which represent NLO correction effect [27]. In Fig. 4, we show the production cross section for scalar boson as a function of its mass with $\sqrt{s}=$ $14 \mathrm{TeV}$ adopting several values of $\sin \alpha\left(\alpha^{\prime}\right)$. We find that a

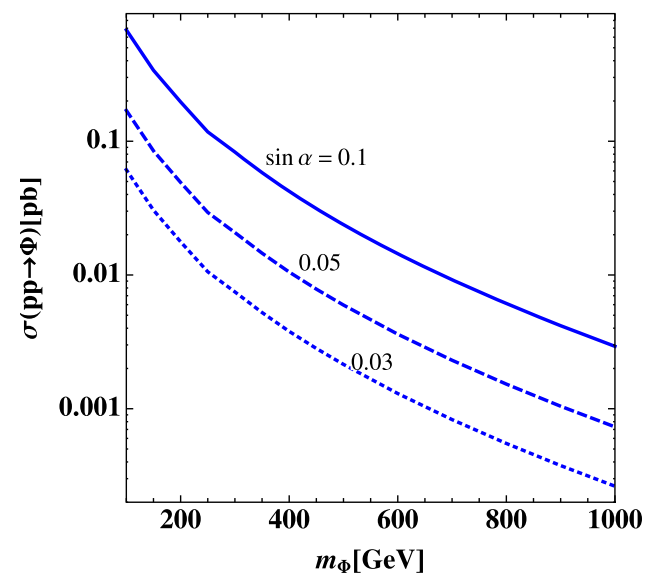

FIG. 4. Cross section for $g g \rightarrow \Phi\left(\Phi=\Phi_{1}, \Phi_{1}^{\prime}\right)$ process as a function of scalar mass with $\sqrt{s}=14 \mathrm{TeV}$. sizable scalar mixing is required to obtain observable cross section. Thus we consider parameter region of $2 m_{X_{1}}>m_{h}$ in our discussion of collider physics since the scalar mixing is constrained for $2 m_{X_{1}}<m_{h}$ as shown in previous subsection.

\section{Branching ratio of extra particles}

Here we estimate BRs of particles in dark sector. The decay widths for the $\Phi_{1}\left[\Phi_{1}^{\prime}\right] \rightarrow \chi_{a} \chi_{b}(a(b)=l, h)$ processes are given by

$$
\begin{aligned}
& \Gamma_{\Phi_{1}\left[\Phi_{1}^{\prime}\right] \rightarrow \chi_{a} \chi_{b}}=\frac{\left|Y_{\Phi_{1}\left[\Phi_{1}^{\prime}\right]}^{\chi_{a} \chi_{b}}\right|^{2}}{8 \pi} m_{\Phi_{1}\left[\Phi_{1}^{\prime}\right]} \lambda^{\frac{1}{2}}\left(m_{\Phi_{1}\left[\Phi_{1}^{\prime}\right]}, m_{\chi_{a}}, m_{\chi_{b}}\right) \\
& \times\left[1-\frac{\left(m_{\chi_{a}}+m_{\chi_{b}}\right)^{2}}{\left.m_{\Phi_{1}\left[\Phi_{1}^{\prime}\right]}^{2}\right]}\right. \\
& Y_{\Phi_{1}}^{\chi_{1} \chi_{l}}=\frac{y_{\chi \phi} \cos \alpha}{2} \cos 2 \theta_{\chi}, \\
& Y_{\Phi_{1}}^{\chi_{h} \chi_{h}}=-\frac{y_{\chi \phi} \cos \alpha}{2} \cos 2 \theta_{\chi}, \\
& Y_{\Phi_{1}}^{\chi i \chi_{h}}=-\frac{y_{\chi \phi} \cos \alpha}{2} \sin 2 \theta_{\chi}, \\
& Y_{\Phi_{1}^{\prime}}^{\chi_{1} \chi_{l}}=\frac{y_{\chi \phi^{\prime}} \cos \alpha^{\prime}}{2} \sin 2 \theta_{\chi}, \\
& Y_{\Phi_{1}^{\prime}}^{\chi_{h} \chi_{h}}=-\frac{y_{\chi \phi} \cos \alpha^{\prime}}{2} \sin 2 \theta_{\chi} \text {, } \\
& Y_{\Phi_{1}}^{\chi_{1} \chi_{h}}=-\frac{y_{\chi \phi} \cos \alpha^{\prime}}{2} \cos 2 \theta_{\chi}, \\
& \lambda\left(m_{1}, m_{2}, m_{3}\right) \equiv 1+\frac{m_{2}^{4}}{m_{1}^{4}}+\frac{m_{3}^{4}}{m_{1}^{4}}-\frac{2 m_{2}^{2}}{m_{1}^{2}}-\frac{2 m_{3}^{2}}{m_{1}^{2}}-\frac{2 m_{2}^{2} m_{3}^{2}}{m_{1}^{4}}
\end{aligned}
$$

where $\Phi_{1}\left[\Phi_{1}^{\prime}\right]$ is mass eigenstate which mixes with the SM Higgs as Eq. (2.16) and (2.17). The dark scalar bosons also decay into dark gauge bosons. For the scenario (1), we obtain 

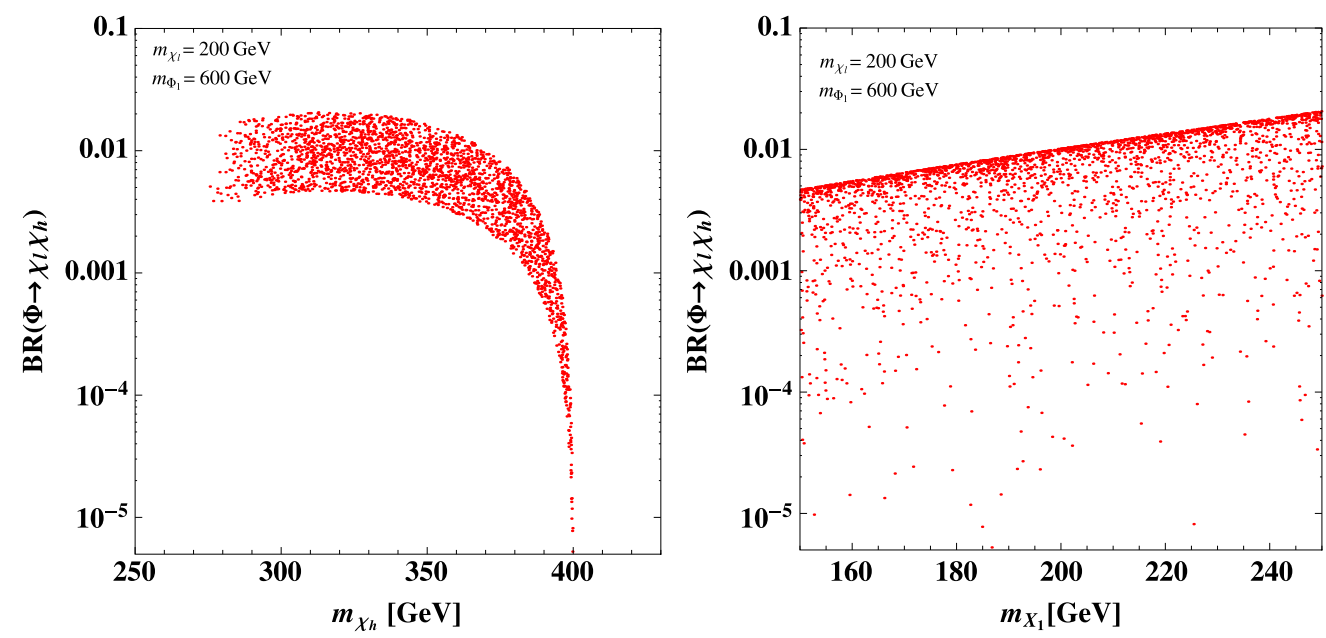

FIG. 5. Branching ratio for $\Phi_{1} \rightarrow \chi_{l} \chi_{h}$ where final state includes both $\bar{\chi}_{l} \chi_{h}$ and $\bar{\chi}_{h} \chi_{l}$.

$$
\begin{gathered}
\Gamma_{\Phi_{1} \rightarrow X_{1} X_{1}}=\frac{g_{D}^{4} \cos ^{2} \alpha}{8 \pi} \frac{v_{\phi}^{2}}{m_{\Phi_{1}}} \sqrt{1-\frac{4 m_{X_{1}}^{2}}{m_{\Phi_{1}}^{2}}}\left[2+\frac{m_{\Phi_{1}}^{4}}{4 m_{X_{1}}^{4}}\left(1-\frac{2 m_{X_{1}}^{2}}{m_{\Phi_{1}}^{2}}\right)^{2}\right], \\
\Gamma_{\Phi_{1} \rightarrow X_{2} X_{2}}=\frac{g_{D}^{4} \cos ^{2} \alpha}{8 \pi} \frac{v_{\phi}^{2}}{m_{\Phi_{1}}} \sqrt{1-\frac{4 m_{X_{2}}^{2}}{m_{\Phi_{1}}^{2}}}\left[2+\frac{m_{\Phi_{1}}^{4}}{4 m_{X_{2}}^{4}}\left(1-\frac{2 m_{X_{2}}^{2}}{m_{\Phi_{1}}^{2}}\right)^{2}\right] .
\end{gathered}
$$

For the scenario (2), we also obtain

$$
\begin{gathered}
\Gamma_{\Phi_{1}^{\prime} \rightarrow X_{3} X_{3}}=\frac{g_{D}{ }^{4} \cos ^{2} \alpha^{\prime}}{8 \pi} \frac{v_{\phi^{\prime}}^{2}}{m_{\Phi_{1}^{\prime}}} \sqrt{1-\frac{4 m_{X_{3}}^{2}}{m_{\Phi_{1}^{\prime}}^{2}}\left[2+\frac{m_{\Phi_{1}^{\prime}}^{4}}{4 m_{X_{3}}^{4}}\left(1-\frac{2 m_{X_{3}}^{2}}{m_{\Phi_{1}^{\prime}}^{2}}\right)^{2}\right],} \\
\Gamma_{\Phi_{1}^{\prime} \rightarrow X_{2} X_{2}}=\frac{g_{D}{ }^{4} \cos ^{2} \alpha^{\prime}}{8 \pi} \frac{v_{\phi^{\prime}}^{2}}{m_{\Phi_{1}^{\prime}}} \sqrt{1-\frac{4 m_{X_{2}}^{2}}{m_{\Phi_{1}^{\prime}}^{2}}\left[2+\frac{m_{\Phi_{1}^{\prime}}^{4}}{4 m_{X_{2}}^{4}}\left(1-\frac{2 m_{X_{2}}^{2}}{m_{\Phi_{1}^{\prime}}^{2}}\right)^{2}\right] .} .
\end{gathered}
$$

In Fig. 5, we show BR for $\Phi_{1} \rightarrow \chi_{1} \chi_{h}$ as functions of $m_{\chi_{h}}$ and $m_{X_{1}}$ in the scenario (1) where we have scanned coupling as $y_{\chi \phi}\left(g_{D}\right) \in[0.5,2.5]([1.5,2.5])$ and fixed some parameters $\sin \alpha=0.1, \theta_{\chi}=\pi / 4, m_{\chi_{1}}=200 \mathrm{GeV}, m_{X_{2}} \simeq$ $m_{X_{3}}=500 \mathrm{GeV}$ and $m_{\Phi_{1}}=600 \mathrm{GeV}$. We find the BR for $\Phi_{1} \rightarrow \chi_{1} \chi_{h}$ is maximally $1.2 \times 10^{-2}$ and dominant decay mode is the $\Phi_{1} \rightarrow X_{1} X_{1}$ mode where the other modes are suppressed. For the scenario (2), we obtain similar result by replacing $X_{1}$ and $X_{3}$ and the corresponding plot is omitted here. The dominant decay mode of $\Phi_{1}$ is summarized in Table II for different mass relations.

\section{Signal at the LHC}

Here we discuss the signature of our model at the LHC based on decay modes of extra scalar bosons which are produced through gluon fusion process via scalar mixing. As we discuss in Sec. IV A, scalar mixing cannot be sizable when the SM Higgs decays into dark gauge bosons. Thus dark gauge boson masses are assumed to be heavier than half of Higgs mass to realize observable signals from extra scalar production.

\begin{tabular}{|c|c|c|c|}
\hline Mass relation & $m_{\Phi_{1}}>2 m_{X_{1}}, m_{X_{3}}>m_{X_{1}}$ & $m_{\Phi_{1}}>2 m_{X_{1}}, m_{X_{1}} \sim m_{X_{3}}$ & $m_{\Phi_{1}}<2 m_{X_{1,3}}, m_{\Phi_{1}}>m_{\chi_{l}}+m_{\chi_{h}}$ \\
\hline & $X_{1} X_{1}$ & $X_{1} X_{1}, X_{2} X_{2}, X_{3} X_{3}$ & $\bar{\chi}_{l} \chi_{h}\left(\bar{\chi}_{h} \chi_{l}\right)$ \\
\hline
\end{tabular}
We summarize possible signature of the model in the following.

TABLE II. Dominant decay mode of $\Phi_{1}$ for some mass relations. 
TABLE III. Cross sections for signal processes in some benchmark points (BPs) for scenario (1) at the LHC 14 TeV. The numbers in front of $\bar{f}_{\mathrm{SM}} f_{\mathrm{SM}}$ indicate number of SM fermion anti-fermion pair in final states.

\begin{tabular}{lccc}
\hline \hline BP & Parameters & Final states & $\sigma B R$ \\
\hline 1 & $\left\{m_{\Phi}, m_{\chi_{l}}, m_{\chi_{h}}, m_{X_{1}}, m_{X_{3}}\right\}=\{800,300,401,200,500\}[\mathrm{GeV}]$ & $2 \bar{f}_{\mathrm{SM}} f_{\mathrm{SM}}$ & $\sim 6[\mathrm{fb}]$ \\
& $\left\{g_{D}, y_{\chi \phi}, y_{\chi \phi^{\prime}}, \sin \alpha\right\}=\{0.4,0.1,0.1,0.1\}$ & $\bar{\chi} \chi_{1} \bar{f}_{\mathrm{SM}} f_{\mathrm{SM}}$ & $\sim 0.01[\mathrm{fb}]$ \\
2 & $\left\{m_{\Phi}, m_{\chi_{l}}, m_{\chi_{h}}, m_{X_{1}}, m_{X_{2}}\right\}=\{600,200,335,200,200\}[\mathrm{GeV}]$ & $2 \bar{f}_{\mathrm{SM}} f_{\mathrm{SM}}$ & $\sim 12.8[\mathrm{fb}]$ \\
& $\left\{g_{D}, y_{\chi \phi}, y_{\chi \phi^{\prime}}, \sin \alpha\right\}=\{0.7,0.1,0.1,0.1\}$ & $4 \bar{f}_{\mathrm{SM}} f_{\mathrm{SM}}$ & $\sim 1.6[\mathrm{fb}]$ \\
3 & $\left\{m_{\Phi}, m_{\chi_{l}}, m_{\chi_{h}}, m_{X_{1}}, m_{X_{2}}\right\}=\{300,75,125,150,200\}[\mathrm{GeV}]$ & $\bar{\chi} \chi_{l} \bar{f}_{\mathrm{SM}} f_{\mathrm{SM}}$ & $\sim 83[\mathrm{fb}]$ \\
& $\left\{g_{D}, y_{\chi \phi}, y_{\chi \phi^{\prime}}, \sin \alpha\right\}=\{0.7,0.2,0.2,0.1\}$ & & \\
\hline \hline
\end{tabular}

\section{1. $\Phi_{1}\left[\Phi_{1}^{\prime}\right] \rightarrow X_{1} X_{1}\left[X_{3} X_{3}\right]$ decay mode}

For $m_{X_{1[3]}}<2 m_{\chi l}, X_{1[3]}$ dominantly decays into SM fermions induced by kinetic mixing. The BR of this decay chain of $\Phi_{1}\left(\Phi_{1}^{\prime}\right)$ is dominant when it is kinematically allowed, $m_{\Phi_{1}\left[\Phi_{1}^{\prime}\right]}>2 m_{X_{1[3]}}$, and provide sizable cross section. The most clear signal is four charged lepton final states which can be well tested at the LHC. For $m_{X_{1[3]}}>2 m_{\chi_{l}}, X_{1[3]}$ dominantly decay into DM since SM fermion mode is suppressed by small kinetic mixing. In this case, the final state becomes transverse missing energy and we need additional jet/photon for tagging.

\section{2. $\Phi_{1}\left[\Phi_{1}^{\prime}\right] \rightarrow X_{2} X_{2}$ decay mode}

For $m_{X_{2}}<m_{\chi_{1}}+m_{\chi_{h}}$, our signal is eight SM fermions coming from decay chain of $X_{2} \rightarrow X_{1} X_{3}\left(X_{1,3} \rightarrow \bar{f}_{\mathrm{SM}} f_{\mathrm{SM}}\right)$. The BR of this decay mode of $\Phi_{1}\left(\Phi_{1}^{\prime}\right)$ can be sizable when it is kinematically allowed and masses among dark gauge bosons are not hierarchical. For $m_{X_{2}}>m_{\chi_{l}}+m_{\chi_{h}}, X_{2}$ dominantly decays into $\bar{\chi}_{h} \chi_{l}\left(\bar{\chi}_{l} \chi_{h}\right)$. Then $\chi_{h}$ decays as $\chi_{h} \rightarrow X_{1[3]}^{(*)} \chi_{l}$ where dark gauge boson is off-shell or onshell depending on mass hierarchy. In this case, we obtain signal of four SM fermions with missing transverse momentum from the decay chain.

\section{3. $\Phi_{1}\left[\Phi_{1}^{\prime}\right] \rightarrow \chi_{L} \chi_{h}$ decay mode}

In this case our signal is SM fermion pair with missing transverse momentum coming from decay chain of $\chi_{h} \rightarrow X_{1,3} \chi_{l}\left(X_{1,3} \rightarrow \bar{f}_{\mathrm{SM}} f_{\mathrm{SM}}\right)$. For $m_{\Phi_{1}\left[\Phi_{1}^{\prime}\right]}>2 m_{X_{1[3]}}$, the BR for the decay mode is small as we discussed above but we can still obtain $\sim 30$ events when $\sigma\left(g g \rightarrow \Phi_{1}\left[\Phi_{1}^{\prime}\right]\right)=$ $10 \mathrm{fb}, \operatorname{BR}\left(\Phi_{1}\left[\Phi_{1}^{\prime}\right] \rightarrow \chi_{1} \chi_{h}\right) \sim 10^{-2}$ and integrated luminosity is $L=300 \mathrm{fb}^{-1}$. On the other hand, for $m_{\Phi_{1}\left[\Phi_{1}^{\prime}\right]}<$ $2 m_{X_{1[3]}}$, the BR of this decay chain is dominant and we can obtain cross section as large as $\Phi_{1}\left[\Phi_{1}^{\prime}\right]$ production cross section.

We indicate dominant decay mode of $\Phi_{1}$ for some mass relations in scenario (1) where we can obtain similar results in scenario (3) interchanging the role of $\left\{\Phi_{1}, X_{1}\right\}$ and $\left\{\Phi_{1}^{\prime} X_{3}\right\}$. In Table III, we show cross sections of signal processes for some benchmark points (BPs) where these parameters can be consistent with relic density of DM. We thus find that signal cross sections can be sizable when scalar mixing is not too small, and signatures of our model at the collider experiments depend on mass relation in dark sector.

Here we briefly discuss experimental constraints. Signatures from neutralino $\chi^{0}$ production followed by decay $\chi^{0} \rightarrow \tilde{G} Z$ ( $\tilde{G}$ gravitino) is similar to our signature from $\chi_{h} \chi_{l}$ production. The upper limit of neutralino production cross section is $\sim 1 \mathrm{pb}$ at LHC $13 \mathrm{TeV}$ when its mass is $125 \mathrm{GeV}$ [28] and the value is sufficiently larger than our cross section for BP3. Cross sections for $2 f_{\text {SM }}$ mode in BP1 and BP2 would be also safe from current data; upper limit of cross sections for $p p \rightarrow$ scalar $\rightarrow Z Z$ at LHC $13 \mathrm{TeV}$ are larger than our values (see Fig. 10 in Ref. [29]).

Among the modes in our BPs, leptonic final states will be the most clear signature. The cross sections are $\sigma \mathrm{BR}\left(2 \ell^{+} \ell^{-}\right) \simeq 0.75 \mathrm{fb}$ for $\mathrm{BP} 1, \sigma \mathrm{BR}\left(2 \ell^{+} \ell^{-}\right) \simeq$ $1.6 \mathrm{fb}$ and $\sigma \mathrm{BR}\left(4 \ell^{+} \ell^{-}\right) \simeq 0.025 \mathrm{fb}$ for $\mathrm{BP} 2$, and $\sigma \mathrm{BR}\left(\bar{\chi}_{1} \chi_{l} \ell^{+} \ell^{-}\right) \simeq 29 \mathrm{fb}$ for $\mathrm{BP} 3$. The cross sections for possible backgrounds in the SM are estimated using MadGraph 5 [30] as follows: $\sigma\left(p p \rightarrow 2 \ell^{+} \ell^{-}\right)=24 \mathrm{fb}$; $\sigma\left(p p \rightarrow \ell^{+} \ell^{-} \nu \bar{\nu}\right)=62(5.1) \mathrm{fb}$ with missing transverse energy cut $\mathscr{E}_{T}>50(150) \mathrm{GeV}$ (cross section of $4 \ell^{+} \ell^{-}$ final state is very small). Thus we can test our model at the LHC and the HL-LHC combining analysis of these processes imposing appropriate kinematical cuts. Further analysis with detailed simulation is beyond the scope of this work and it is left for future work.

\section{SUMMARY AND DISCUSSIONS}

We have discussed a model of dark sector described by $S U(2)_{D}$ gauge symmetry in which two triplet real scalar fields and one doublet Dirac fermion are introduced. In our scenario, $S U(2)_{D}$ symmetry is broken to discrete $Z_{2}$ symmetry by VEVs of two triplet scalar fields. Then remaining $Z_{2}$ symmetry guarantees stability of DM candidate which is the lighter component from doublet fermion $\chi_{l}$. In the gauge sector, we consider kinetic mixing term between $S U(2)_{D}$ and $U(1)_{Y}$ which is assumed to be generated via 5-dimensional operators. Then we have investigated dark gauge sector which provides three 
massive dark gauge bosons $X_{1,2,3}$, two of which can mix with SM gauge boson via the kinetic mixings.

We have estimated relic density of our DM candidate where the observed value is explained via gauge interactions in dark sector with kinetic mixing effect as a portal to the SM sector. Then we have explored the parameter region satisfying observed relic density. We have found that the relic density is explained by the process, $\chi_{1} \bar{\chi}_{l} \rightarrow X_{1,2,3} X_{1,2,3}$, in large parameter region while we need fine tuning to obtain resonant enhancement for the process, $\chi_{1} \bar{\chi}_{l} \rightarrow f_{\mathrm{SM}} \bar{f}_{\mathrm{SM}}$, via dark gauge boson exchange with kinetic mixing.

Implications to collider physics have been discussed such as $h \rightarrow Z^{\prime} Z^{\prime}$ decay, and extra scalar production and its possible signals at the LHC. We have found that the constraint from $h \rightarrow Z^{\prime} Z^{\prime}$ branching ratio restricts scalar mixing with the SM Higgs and $S U(2)_{D}$ gauge coupling severely when the mode is kinematically allowed. Extra scalar boson can be produced by gluon fusion process through scalar mixing associated with the SM Higgs. For extra scalar production, we obtain some specific signatures depending on mass relation of dark sector particles.

\section{ACKNOWLEDGMENTS}

The work is supported in part by KIAS Individual Grants, Grants No. PG021403 (PK) and No. PG054702 (TN) at Korea Institute for Advanced Study, and by National Research Foundation of Korea (NRF) Grant No. NRF-2019R1A2C3005009 (PK), funded by the Korea government (MSIT). This research was supported by an appointment to the JRG Program at the APCTP through the Science and Technology Promotion Fund and Lottery Fund of the Korean Government. This was also supported by the Korean Local GovernmentsGyeongsangbuk-do Province and Pohang City (H. O.). H.O. is sincerely grateful for KIAS and the members who provided me with huge hospitality during my stay.
[1] P. Ko, J. Korean Phys. Soc. 73, 449 (2018).

[2] L. M. Krauss and F. Wilczek, Phys. Rev. Lett. 62, 1221 (1989).

[3] H. Zhang, C. S. Li, Q. H. Cao, and Z. Li, Phys. Rev. D 82, 075003 (2010).

[4] C. W. Chiang, T. Nomura, and J. Tandean, J. High Energy Phys. 01 (2014) 183.

[5] C. H. Chen and T. Nomura, Phys. Lett. B 746, 351 (2015).

[6] C. H. Chen and T. Nomura, Phys. Rev. D 93, 074019 (2016).

[7] C. H. Chen, C. W. Chiang, and T. Nomura, Phys. Rev. D 97, 061302 (2018).

[8] C. Gross, O. Lebedev, and Y. Mambrini, J. High Energy Phys. 08 (2015) 158.

[9] T. Hambye, J. High Energy Phys. 01 (2009) 028.

[10] C. Boehm, M. J. Dolan, and C. McCabe, Phys. Rev. D 90, 023531 (2014).

[11] S. Baek, P. Ko, and W. I. Park, J. Cosmol. Astropart. Phys. 10 (2014) 067.

[12] V. V. Khoze and G. Ro, J. High Energy Phys. 10 (2014) 61.

[13] R. Daido, S. Y. Ho, and F. Takahashi, J. High Energy Phys. 01 (2020) 185.

[14] H. Davoudiasl and I. M. Lewis, Phys. Rev. D 89, 055026 (2014).

[15] A. Karam and K. Tamvakis, Phys. Rev. D 92, 075010 (2015).

[16] S. Choi, S. Jung, and P. Ko, J. High Energy Phys. 10 (2013) 225.
[17] K. Cheung, P. Ko, J. S. Lee, and P. Y. Tseng, J. High Energy Phys. 10 (2015) 057.

[18] C. A. Argüelles, X. G. He, G. Ovanesyan, T. Peng, and M. J. Ramsey-Musolf, Phys. Lett. B 770, 101 (2017).

[19] E. J. Weinberg, Classical Solutions in Quantum Field Theory (Cambridge University Press, Cambridge, England, 2012).

[20] G. Belanger, F. Boudjema, A. Pukhov, and A. Semenov, Comput. Phys. Commun. 192, 322 (2015).

[21] E. Aprile et al. (XENON Collaboration), Phys. Rev. Lett. 121, 111302 (2018).

[22] X. Cui et al. (PandaX-II Collaboration), Phys. Rev. Lett. 119, 181302 (2017).

[23] M. Aaboud et al. (ATLAS Collaboration), J. High Energy Phys. 06 (2018) 166.

[24] J. F. Gunion, H. E. Haber, G. L. Kane, and S. Dawson, Front. Phys. 80, 1 (2000).

[25] A. Belyaev, N. D. Christensen, and A. Pukhov, Comput. Phys. Commun. 184, 1729 (2013).

[26] P. M. Nadolsky, H. L. Lai, Q. H. Cao, J. Huston, J. Pumplin, D. Stump, W. K. Tung, and C.-P. Yuan, Phys. Rev. D 78, 013004 (2008).

[27] A. Djouadi, Phys. Rep. 457, 1 (2008).

[28] A. M. Sirunyan et al. (CMS Collaboration), arXiv:2012 .08600 .

[29] A. M. Sirunyan et al. (CMS Collaboration), J. High Energy Phys. 06 (2018) 127; 03 (2019) 128(E).

[30] J. Alwall, R. Frederix, S. Frixione, V. Hirschi, F. Maltoni, O. Mattelaer, H. S. Shao, T. Stelzer, P. Torrielli, and M. Zaro, J. High Energy Phys. 07 (2014) 079. 